\title{
On Nonlocal Monotone Difference Schemes for Scalar Conservation Laws*
}

\author{
By Bradley J. Lucier
}

\begin{abstract}
We provide error analyses for explicit, implicit, and semi-implicit monotone finitedifference schemes on uniform meshes with nonlocal numerical fluxes. We are motivated by finite-difference discretizations of certain long-wave (Sobolev) regularizations of the conservation laws that explicitly add a dispersive term as well as a nonlinear dissipative term. We also develop certain relationships between dispersion and stability in finite-difference schemes. Specifically, we find that discretization and explicit dispersion have identical effects on the amount of artificial dissipation necessary for stability.
\end{abstract}

1. Introduction. We analyze a class of monotone numerical methods for the approximate solution of the hyperbolic conservation laws

$$
\begin{gathered}
u_{t}+f(u)_{x}=0, \quad x \in \mathbf{R}, t \in(0, T], \\
u(x, 0)=u_{0}(x), \quad x \in \mathbf{R} .
\end{gathered}
$$

We give convergence results with error estimates for explicit, implicit, and semi-implicit finite-difference schemes on uniform meshes with nonlocal numerical fluxes. The motivating examples for these methods are finite-difference discretizations of a Sobolev-type regularization of $(\mathrm{C})$,

$$
u_{t}+f(u)_{x}-\nu g(u)_{x x}-\alpha^{2} u_{x x t}=0 .
$$

Equation (S), which regularizes $(\mathrm{C})$ by adding a term simulating dispersive effects $\left(-\alpha^{2} u_{x x t}\right)$ as well as dissipation $\left(-\nu g(u)_{x x}\right)$, has been studied in [20] as a singular perturbation of $(C)$; one can find other references there. For example, the implicit difference scheme that we consider is

$$
d_{t} U_{i}^{n}+d_{x} f\left(U^{n+1}\right)_{i}-\nu d_{x}^{2} g\left(U^{n+1}\right)_{i}-\alpha^{2} d_{x}^{2} d_{t} U_{i}^{n}=0, \quad i \in \mathbf{Z}, n \geqslant 0,
$$

where $d_{x} W_{i}^{n}=\left(W_{i+1}^{n}-W_{i-1}^{n}\right) / 2 h, d_{x}^{2} W_{i}^{n}=\left(W_{i+1}^{n}-2 W_{i}^{n}+W_{i-1}^{n}\right) / h^{2}$, and $d_{t} W_{i}^{n}$ $=\left(W_{i}^{n+1}-W_{i}^{n}\right) / \Delta t$, for any mesh function $W$. The positive parameters $h$ and $\Delta t$ are the mesh size and the time step, respectively. Such methods are similar to finite-difference and finite-element schemes introduced by Douglas et al. [8], and to artificial time methods, introduced by Jameson and Baker [14], for finding steadystate solutions of the Euler equations.

Received August 25, 1982; revised October 23, 1983 and June 3, 1985.

1980 Mathematics Subject Classification. Primary 35B50, 35Q20, 65M10.

*This research was sponsored by the U. S. Army under contract DAAG29-80-C-0041. This material is based upon work partially supported by the National Science Foundation under grant MCS-7927062, Mod. 1. 
In Section 3, we study the stability in $L^{1}(\mathbf{Z})$ of the above difference scheme for various values of $\alpha$ and $\nu$. This exercise illuminates the relationship between dispersion and stability of several finite-difference schemes for (C). Specifically, we find that discretization and explicit dispersion have identical effects on the amount of artificial dissipation necessary for stability.

It is well known that classical smooth solutions of (C) do not exist in general, and that weak solutions, satisfying the equation

$$
\iint_{\mathbf{R} \times[0, t]}\left(u \phi_{t}+f(u) \phi_{x}\right) d x d t+\int_{\mathbf{R}} u_{0}(x) \phi(x, 0) d x=0
$$

for all continuously differentiable $\phi$ with support in $\mathbf{R} \times(-\infty, T]$, are not unique (see, for example, [26]). Existence and uniqueness results may be provided for certain classes of weak solutions of (C) through the prescription of an extra condition, known as an entropy condition. The theory for solutions of $(C)$ used in this paper is expressed in the following theorem.

THEOREM 1.1 [KRUZHKOV]. If $f$ is locally Lipschitz continuous, then for any $u_{0} \in B V(\mathbf{R})$ and for any $T>0$ there is a unique $u \in B V(\mathbf{R} \times[0, T]) \cap C^{0}([0, T]$, $\left.L_{\text {loc }}^{1}(\mathbf{R})\right)$ such that $u$ satisfies (1.2) and, in addition, satisfies the entropy condition: For all $\phi \in C_{0}^{1}(\mathbf{R} \times[0, T])$, with $\phi \geqslant 0$, and for all $c \in \mathbf{R}$,

$$
\iint_{\mathbf{R} \times[0, T]}\left[|u-c| \phi_{t}+\operatorname{sgn}(u-c)(f(u)-f(c)) \phi_{x}\right] d x d t \geqslant 0 .
$$

(This theorem has somewhat weaker hypotheses and a stronger conclusion than Kruzhkov presented in [15]; it may be proved using techniques found in [20] and Kuznetsov's approximation theory presented below.)

Kuznetsov [16] proposed a general theory of approximation for solutions of (C) in an arbitrary number of spatial dimensions. We formulate the one-dimensional version as follows.

THEOREM 1.2. Let $u$ be an entropy solution of (C) with $u_{0} \in B V(\mathbf{R})$, and let $v: \mathbf{R}^{+} \rightarrow L_{\text {loc }}^{1}(\mathbf{R})$ have left and right limits for any $t$, and be right continuous. Pick $a$ positive, symmetric function $\eta(\xi)$ with support in $[-1,1]$ and integral 1 , positive numbers $\varepsilon$ and $\varepsilon_{0}$, and let

$$
\omega(x, t)=\frac{1}{\varepsilon_{0}} \eta\left(\frac{t}{\varepsilon_{0}}\right) \frac{1}{\varepsilon} \eta\left(\frac{x}{\varepsilon}\right) .
$$

Define the "Kruzhkov form"

$$
\begin{aligned}
\Lambda_{t}^{\epsilon_{0}, \varepsilon}=\int_{S \times S} & {\left[\left|v\left(x^{\prime \prime}, t^{\prime \prime}\right)-u\left(x^{\prime}, t^{\prime}\right)\right| \frac{\partial}{\partial t^{\prime \prime}} \omega\left(x^{\prime \prime}-x^{\prime}, t^{\prime \prime}-t^{\prime}\right)\right.} \\
& +\operatorname{sgn}\left(v\left(x^{\prime \prime}, t^{\prime \prime}\right)-u\left(x^{\prime}, t^{\prime}\right)\right)\left(f\left(v\left(x^{\prime \prime}, t^{\prime \prime}\right)\right)-f\left(u\left(x^{\prime}, t^{\prime}\right)\right)\right) \\
& \left.\times \frac{\partial}{\partial x^{\prime \prime}} \omega\left(x^{\prime \prime}-x^{\prime}, t^{\prime \prime}-t^{\prime}\right)\right] d x^{\prime \prime} d t^{\prime \prime} d x^{\prime} d t^{\prime} \\
+ & \int_{S \times \mathbf{R}}\left[\omega\left(x^{\prime \prime}-x^{\prime}, 0-t^{\prime}\right)\left|v\left(x^{\prime \prime}, 0\right)-u\left(x^{\prime}, t^{\prime}\right)\right|\right. \\
& \left.-\omega\left(x^{\prime \prime}-x^{\prime}, t-t^{\prime}\right)\left|v\left(x^{\prime \prime}, t-0\right)-u\left(x^{\prime}, t^{\prime}\right)\right|\right] d x^{\prime \prime} d x^{\prime} d t^{\prime},
\end{aligned}
$$


where $S=\mathbf{R} \times[0, t]$. Then,

$$
\begin{aligned}
\|u(t)-v(t)\|_{L^{1}(\mathbf{R})} \leqslant & \|u(0)-v(0)\|_{L^{1}(\mathbf{R})}+\left(2 \varepsilon+\|f\|_{\text {Lip }} \varepsilon_{0}\right)\left|u_{0}\right|_{B V(\mathbf{R})} \\
\cdot & +\sup _{\left\{t^{\prime},|\tau|<\varepsilon_{0} \mid-t^{\prime}<\tau<t-t^{\prime}\right\}}\left\|v\left(t^{\prime}+\tau\right)-v\left(t^{\prime}\right)\right\|_{L^{1}(\mathbf{R})}-\Lambda_{t}^{\varepsilon_{0}, \varepsilon} .
\end{aligned}
$$

For most numerical schemes in this paper, $-\Lambda_{t}^{\varepsilon_{0}, \varepsilon}$ will be bounded in such a way that the preceding inequality will simplify to

$$
\|u(t)-v(t)\|_{L^{1}(\mathbf{R})} \leqslant\|u(0)-v(0)\|_{L^{1}(\mathbf{R})}+C\left(h^{1 / 2}|t|^{1 / 2}+h\right)\left|u_{0}\right|_{B V(\mathbf{R})},
$$

where $C$ is a positive constant. Note that for large $t$, the error is bounded by $(t h)^{1 / 2}\left|u_{0}\right|_{B V(\mathbf{R})}$; in many important instances this bound is sharp.

Finite-difference approximations in conservative form for the conservation law (C) were introduced by Lax and Wendroff [18]. Explicit conservative schemes have the form

$$
\begin{aligned}
\frac{U_{i}^{n+1}-U_{i}^{n}}{\Delta t}+\frac{F\left(U_{i-k}^{n}, \ldots, U_{i+k}^{n}\right)-F\left(U_{i-k-1}^{n}, \ldots, U_{i+k-1}^{n}\right)}{h} & =0, \\
& i \in \mathbf{Z}, n \geqslant 0 .
\end{aligned}
$$

The function $F$ is the numerical flux; the scheme is consistent if $F(c, \ldots, c)=f(c)$ for all $c$. Such methods are called monotone if $U^{0}, V^{0} \in B V(\mathbf{Z})$ and $U_{i}^{0} \geqslant V_{i}^{0}$ for all $i \in \mathbf{Z}$ implies that, for all $n \geqslant 1, U_{i}^{n} \geqslant V_{i}^{n}$ for all $i \in \mathbf{Z}$.

Harten et al. [12] proved that if the solutions of monotone, consistent, conservative-form finite-difference approximations to (C) converge as $h \rightarrow 0$, they converge to the entropy solution of (C). Using Theorem 1.2, Kuznetsov [16] proved that monotone schemes for $(\mathrm{C})$ converge to the entropy solution in more than one space dimension, and he provided suitable error estimates. Later, Crandall and Majda [4] proved a similar result without the error estimates; their treatment of the numerical entropy condition is more illuminating than Kuznetsov's, however.

Sanders [22] proved convergence, with error estimates, for certain three-point schemes with fixed nonuniform grid spacings. (Sanders' treatment of Kuznetsov's theory, although correct in outline, erroneously omits the boundary terms in the definition of $\Lambda_{t}^{\varepsilon_{0}, \varepsilon}$.) Douglas [7] and Douglas and Wheeler [9] proved convergence for methods for which their nonuniform spatial grid was changed from one time step to the next under certain constraints. In a series of papers [17], [24], [25], Kuznetsov and Voloshin analyzed schemes similar to those studied here. In comparision with our results, their hypotheses are more stringent, and their results are weaker; for example, the scheme (1.1), when considered as a three-point scheme, does not satisfy Voloshin's definition of an implicit monotone scheme for any positive value of $\alpha$. Because of this, our analysis of (1.1) depends strongly on considering the finite-difference operator to have an infinite stencil (or domain of dependence), so that the numerical flux $F$ maps $B V(\mathbf{Z})$ into $\mathbf{R}$ instead of mapping $\mathbf{R}^{n}$ into $\mathbf{R}$.

We first give some preliminary results and notation. In the second section, the convergence theory for the general equation is presented. In the final section, we show that discretizations of (S) satisfy the hypotheses of our theorems and we discuss the stability of certain difference schemes. 
The mappings $\sigma_{i}$ on $\mathbf{Z}$, the integers, map $j$ to $i+j$, with $\sigma=\sigma_{1}$. An operator $A$ is said to commute with translations if $A(u(\cdot+y))=(A u)(\cdot+y) . L_{\text {loc }}^{1}(\mathbf{R})$, the space of all functions $u$ that are integrable on compact subsets of $\mathbf{R}$, may be considered a vector lattice, with the natural ordering $u \geqslant v$ if $u(x) \geqslant v(x)$ for all $x \in \mathbf{R}$. Possibly nonlinear operators $T$ of a vector lattice to itself that preserve the ordering, so that $u \geqslant v$ implies that $T u \geqslant T v$, are order-preserving, or monotone. (If $T$ is linear, it is called positive.)

We will consider solutions of (S) and (C) that are in $B V\left(\mathbf{R}^{n}\right)$, the set of all functions on $\mathbf{R}^{n}$ whose first distributional derivatives are bounded measures. The $B V$ seminorm of $u$ is given by

$$
|u|_{B V\left(\mathbf{R}^{n}\right)}=\int_{\mathbf{R}^{n}} \sum_{j \leqslant n}\left|\frac{\delta u}{\delta x_{j}}\right| d x .
$$

(See Giusti [11].) The $B V(\mathbf{R})$-norm will be defined as

$$
\|u\|_{B V(\mathbf{R})}=\left|\lim _{x \rightarrow-\infty} u(x)\right|+|u|_{B V(\mathbf{R})} .
$$

$B V(I)$, where $I$ is a bounded interval, and $B V(\mathbf{R} \times I)$ are defined analogously. The $B V(\mathbf{Z})$-seminorm of a function defined on the integers is

$$
|U|_{B V(\mathbf{Z})}=\sum_{j \in \mathbf{Z}}\left|U_{j}-U_{j-1}\right| \text {. }
$$

2. Convergence Results. Our extension of (1.4) is to solve

$$
\frac{U^{n+1}-U^{n}}{\Delta t}+\frac{1}{h}\left\{F_{1}\left(\sigma U^{n+1}\right)+F_{2}\left(\sigma U^{n}\right)-\left(F_{1}\left(U^{n+1}\right)+F_{2}\left(U^{n}\right)\right)\right\}=0
$$

for $n \geqslant 0$, where the initial vector $U^{0}$ is provided in a manner to be described below. The vectors $U^{n}$ and $U^{n+1}$ are in $B V(\mathbf{Z})$, and the functions $F_{1}$ and $F_{2}$ are assumed to satisfy the following assumptions, which seem to be necessary to reach the conclusions presented in the following theorems; also, to analyze (1.1), it seems necessary to consider nonlocal numerical fluxes. We make the following four assumptions about the numerical methods in this section.

Assumption 1. There are two functions $f_{1}, f_{2}: B V(\mathbf{Z}) \rightarrow \mathbf{R}$, and constants $L_{i}$, $i \in \mathbf{Z}$, such that if $U \in B V(\mathbf{Z}), \eta \in \mathbf{R}$, and $l=1$ or 2 , then, for every vector $e^{i}$ with $e_{j}^{i}=\delta_{j}^{i}$

$$
\left|f_{l}\left(U+\eta e^{i}\right)-f_{l}(U)\right| \leqslant L_{i} \eta
$$

and

$$
\sum_{i \in \mathbf{Z}} L_{i}=L<\infty
$$

This assumption ensures that the numerical fluxes $f_{1}$ and $f_{2}$ are Lipschitz continuous in each component, and that the Lipschitz constants are summable at infinity. Any local flux of the form (1.4) will automatically satisfy the decay condition if it is Lipschitz continuous, because $L_{i}=0$ for large enough $i$. The numerical flux $f_{1}$ will be evaluated at the advanced time level, and $f_{2}$ will be evaluated at the current one.

For $l=1$ and 2, define the mappings $F_{l}: B V(\mathbf{Z}) \rightarrow B V(\mathbf{Z})$ by

$$
F_{l}(U)_{i}=f_{l}\left(\sigma_{i} U\right)
$$


and the mappings $A_{l}: B V(\mathbf{Z}) \rightarrow L^{1}(\mathbf{Z})$ by

$$
A_{l}(U)=F_{l}(\sigma U)-F_{l}(U) .
$$

The $L^{1}(\mathbf{Z})$-norm of $A_{l}(U)$ is bounded by $L$ times the $B V(\mathbf{Z})$-seminorm of $U$, for

$$
\begin{aligned}
\left\|F_{l}(\sigma U)-F_{l}(U)\right\|_{L^{\prime}(\mathbf{Z})} & =\sum_{i \in \mathbf{Z}}\left|f_{l}\left(\sigma_{i+1} U\right)-f_{l}\left(\sigma_{i} U\right)\right| \\
& \leqslant \sum_{i \in \mathbf{Z}} \sum_{j \in \mathbf{Z}} L_{j}\left|U_{i+j+1}-U_{i+j}\right| \\
& =\sum_{j \in \mathbf{Z}} L_{j}|U|_{B V(\mathbf{Z})}=L|U|_{B V(\mathbf{Z})} .
\end{aligned}
$$

Similarly, if $U$ and $V$ are in $B V(\mathbf{Z})$, and $U-V \in L^{1}(\mathbf{Z})$, then

$$
\left\|F_{l}(U)-F_{l}(V)\right\|_{L^{1}(\mathbf{Z})} \leqslant L\|U-V\|_{L^{1}(\mathbf{Z})} .
$$

Note that $F_{l}$ and $A_{l}$ commute with translations.

Assumption 2. If $C_{i}=c$ for all $i$, then

$$
f_{1}(C)+f_{2}(C)=f(c) .
$$

Thus, the numerical fluxes are consistent with $f$.

Assumption 3. For any $U, V \in B V(\mathbf{Z})$ with $U-V \in L^{1}(\mathbf{Z})$,

$$
\sum_{i \in \mathbf{Z}}\left(A_{1}(U)_{i}-A_{1}(V)_{i}\right) \operatorname{sgn}\left(U_{i}-V_{i}\right) \geqslant 0 .
$$

We will show that this condition implies that the solution operator of the implicit equation $U^{n+1}+\Delta t A_{1}\left(U^{n+1}\right) / h=U^{n}$ is order-preserving on $B V(\mathbf{Z})$.

AssUMPTION 4. The mapping

$$
H_{2}(U)=U-\frac{\Delta t}{h}\left\{F_{2}(\sigma U)-F_{2}(U)\right\}
$$

is order-preserving on $B V(\mathbf{Z})$.

The following theorem deals with the question of the existence and uniqueness of the solution of the system (2.1).

THEOREM 2.1. If $f_{1}, f_{2}, h$ and $\Delta t$ are given such that Assumptions 1 through 4 are satisfied, then for any $U \in B V(\mathbf{Z})$ there is a unique $U^{*} \in B V(\mathbf{Z})$ that satisfies

(a) $\left(U^{*}-U\right) / \Delta t+\left\{F_{1}\left(\sigma U^{*}\right)+F_{2}(\sigma U)-\left(F_{1}\left(U^{*}\right)+F_{2}(U)\right)\right\} / h=0$.

Furthermore,

(b) $\left|U^{*}\right|_{B V(\mathbf{Z})} \leqslant|U|_{B V(\mathbf{Z})}$,

(c) $\left\|U^{*}-U\right\|_{L^{1}(\mathbf{Z})} \leqslant 2 L \Delta t|U|_{B V(\mathbf{Z})} / h$

(d) $\sum_{i \in \mathbf{Z}}\left(U_{i}^{*}-U_{i}\right) h=\Delta t\left(f\left(U^{-}\right)-f\left(U^{+}\right)\right)$, where $U^{+}=\lim _{j \rightarrow \infty} U_{j}$ and $U^{-}=$ $\lim _{i \rightarrow-\infty} U_{j}$,

(e) if $U_{i} \geqslant V_{i}$ for all $i \in \mathbf{Z}$, then $U_{i}^{*} \geqslant V_{i}^{*}$ for all $i \in \mathbf{Z}$, and

(f) for all $i \in \mathbf{Z}, \inf _{j \in \mathbf{Z}} U_{j} \leqslant U_{i}^{*} \leqslant \sup _{j \in \mathbf{Z}} U_{j}$.

The following two lemmas are used in the proof of Theorem 2.1.

Lemma 2.1 (Crandall-TaRTaR [5]). If $T: L^{1}(\Omega) \rightarrow L^{1}(\Omega)$ for some measure space $(\Omega, d \mu)$ and $\int_{\Omega} T u d \mu=\int_{\Omega} u d \mu$, then $T$ is a contraction on $L^{1}(\Omega)$ if and only if $T$ is order-preserving. 
LEMMA 2.2 [20]. If $T$ maps $L^{1}(\mathbf{Z})$ or $L^{1}(\mathbf{R})$ to itself, preserves the integral, and commutes with translations, then $T$ satisfies a maximum and minimum principle, that is,

$$
\lim \sup T u(x) \leqslant \lim \sup u(x)
$$

and

$$
\liminf T u(x) \geqslant \liminf u(x)
$$

Proof of Theorem 2.1. We will at times use the functional notation $H(U)$ for $U^{*}$. Solutions of (a) will be constructed first for $U \in L^{1}(\mathbf{Z})$.

Rewrite (a) as

$$
U^{*}+\frac{\Delta t}{h}\left\{F_{1}\left(\sigma U^{*}\right)-F_{1}\left(U^{*}\right)\right\}=U-\frac{\Delta t}{h}\left\{F_{2}(\sigma U)-F_{2}(U)\right\},
$$

or

$$
U^{*}+\frac{\Delta t}{h} A_{1}\left(U^{*}\right)=U-\frac{\Delta t}{h} A_{2}(U) \equiv H_{2}(U) .
$$

Let $H_{1}(V)$ denote the solution operator of

$$
H_{1}(V)+\frac{\Delta t}{h}\left\{F_{1}\left(\sigma H_{1}(V)\right)-F_{1}\left(H_{1}(V)\right)\right\}=V .
$$

Then $H(U)=H_{1} \circ H_{2}(U)$. Since $\sum_{i \in \mathbf{Z}} A_{l}(U)_{i}=0$ if $U \in L^{1}(\mathbf{Z})$,

$$
\sum_{i \in \mathbf{Z}} H_{1}(U)_{i}=\sum_{i \in \mathbf{Z}} H_{2}(U)_{i}=\sum_{i \in \mathbf{Z}} U_{i}
$$

Thus, $H_{1}, H_{2}$, and $H$ preserve the integral. Similarly, since $A_{1}$ and $A_{2}$ commute with translations, $H_{1}, H_{2}$, and $H$ commute with translations.

Assumption 3, together with the fact that $A_{1}$ is continuous, implies that $A_{1}$ is $m$-accretive on $L^{1}(\mathbf{Z})$ (see [6]), so that the Crandall-Liggett theorem [3] implies that $H_{1}$ exists and is a contraction on $L^{1}(\mathbf{Z})$. By Lemma 2.1, $H_{1}$ is also order-preserving.

Because of Assumption 4, $\mathrm{H}_{2}$ is order-preserving. Thus, by Lemma 2.1 again, $H=H_{1} \circ H_{2}$ is an order-preserving contraction that preserves the integral and commutes with translations. Using these properties, it is easy to show that

$$
\|H(U)\|_{B V(\mathbf{Z})} \leqslant\|U\|_{B V(\mathbf{Z})},
$$

and Lemma 2.2 then implies that for all $i \in \mathbf{Z}$,

$$
\inf _{j \in \mathbf{Z}} U_{j} \leqslant H(U)_{i} \leqslant \sup _{j \in \mathbf{Z}} U_{j}
$$

As for (c), notice that

$$
\begin{aligned}
\left\|U^{*}-U\right\|_{L^{1}(\mathbf{Z})} & =\frac{\Delta t}{h}\left\|A_{1}\left(U^{*}\right)+A_{2}(U)\right\|_{L^{1}(\mathbf{Z})} \\
& \leqslant L \frac{\Delta t}{h}\left(\left|U^{*}\right|_{B V(\mathbf{Z})}+|U|_{B V(\mathbf{Z})}\right) \leqslant 2 L \frac{\Delta t}{h}|U|_{B V(\mathbf{Z})} .
\end{aligned}
$$

We have now shown that $U^{*}$ exists if $U \in L^{1}(\mathbf{Z})$; it remains to show that $U^{*}$ exists for any $U \in B V(\mathbf{Z})$ with the properties (b) through (f). For any $U \in B V(\mathbf{Z})$, let $U^{n}=\chi_{[-n, n]} \cdot U .\left(U^{n}\right.$ takes the same values as $U$ inside $[-n, n]$, but is 0 outside this interval.) Then $U^{n} \in L^{1}(\mathbf{Z}),\left\|U^{n}\right\|_{L^{\infty}(\mathbf{Z})} \leqslant\|U\|_{L^{\infty}(\mathbf{Z})}$, and $\left\|U^{n}\right\|_{B V(\mathbf{Z})} \leqslant\|U\|_{B V(\mathbf{Z})}+$ $2\|U\|_{L^{x}(\mathbf{Z})}$ independently of $n$. Thus, as $n$ tends to infinity, $H\left(U^{n}\right)$ is uniformly bounded in $B V(\mathbf{Z})$ and $L^{\infty}(\mathbf{Z})$. Therefore, there is a subsequence of the $U^{n}$, renamed 
$U^{n}$, and a $U^{*}$ such that $H\left(U^{n}\right) \rightarrow U^{*}$ in $L_{\text {loc }}^{1}(\mathbf{Z})$, i.e., uniformly on any bounded interval. We omit a series of tedious arguments, which use the fact that the Lipschitz constants of $f_{1}$ and $f_{2}$ are summable, that show that the limiting function $U^{*}$ is unique, and satisfies (a) through (f). The argument, which is similar in spirit to the proof of Lemma 2.3, may be found in the technical report [19].

In the sequel we will use the characteristic functions

$$
\chi_{i}^{n}(x, t)= \begin{cases}1 & \text { if }(x, t) \in[(i-1 / 2) h,(i+1 / 2) h) \times[n \Delta t,(n+1) \Delta t) \\ 0 & \text { otherwise. }\end{cases}
$$

The following theorem is our main result.

THEOREM 2.2. Let $h, \Delta t>0$ be such that the ratio $\Delta t / h=\lambda$ is fixed. Assume that $u_{0} \in B V(\mathbf{R})$, and let $f_{1}$ and $f_{2}$ be given such that Assumptions 1 through 4 are satisfied. Let $U_{h}=\left\{U_{i}^{n}\right\}_{i \in \mathbf{Z}}^{n>0}$ be defined by

$$
\begin{array}{r}
\frac{U^{n+1}-U^{n}}{\Delta t}+\frac{1}{h}\left\{F_{1}\left(\sigma U^{n+1}\right)-F_{1}\left(U^{n+1}\right)+F_{2}\left(\sigma U^{n}\right)-F_{2}\left(U^{n}\right)\right\}=0 \\
\text { for } n \geqslant 0,
\end{array}
$$

and

$$
U_{i}^{0}=\frac{1}{h} \int_{(i-1 / 2) h}^{(i+1 / 2) h} u_{0}(x) d x .
$$

Then, for any $T>0$, the functions

$$
\tilde{U}_{h}=\sum_{\substack{i \in \mathbf{Z} \\ n \Delta t \leqslant T}} U_{i}^{n} \chi_{i}^{n}
$$

converge to the entropy solution of $(\mathrm{C})$ in $L_{\text {loc }}^{1}(\mathbf{R})$ as $h \rightarrow 0$. If , in addition, we assume that

$$
\sum_{j \in \mathbf{Z}}|j| L_{j}=M<\infty
$$

then for any $0 \leqslant t \leqslant T, \tilde{U}_{h}$ satisfies

$$
\left\|\tilde{U}_{h}(t)-u(t)\right\|_{L^{1}(\mathbf{R})} \leqslant C\left(h+(h t)^{1 / 2}\right)\left|u_{0}\right|_{B V(\mathbf{R})},
$$

where $u$ is the entropy solution of $(\mathrm{C})$, and $C$ depends on $L, \Delta t / h$, and $M$.

To prove this result, we will use the following lemma.

Lemma 2.3. Let $f_{1}$ and $f_{2}$ be given such that Assumptions 1 and 2 hold,

$$
V_{h}=\left\{V_{h k}^{n}\right\}_{k \in \mathbf{Z}}^{n>0}, \quad \tilde{V}_{h}=\sum_{\substack{k \in \mathbf{Z} \\ n \Delta t \leqslant T}} V_{h k}^{n} \chi_{k}^{n} \in L_{\mathrm{loc}}^{1}(\mathbf{R} \times[0, T]),
$$

and let there be a constant $C$ such that for all $h, n \geqslant 0$,

$$
\left|V_{h}^{n}\right|_{B V(\mathbf{Z})}+\left\|V_{h}^{n}\right\|_{L^{\infty}(\mathbf{Z})} \leqslant C .
$$

If $\tilde{V}_{h} \rightarrow v$ in $L_{\mathrm{loc}}^{1}(\mathbf{R} \times[0, T])$ as $h \rightarrow 0, l=1$ or 2 , and

$$
\tilde{f}_{h}=\sum_{\substack{i \in \mathbf{Z} \\ n \Delta t \leqslant T}} F_{l}\left(V_{h}^{n}\right)_{i} \chi_{i}^{n}
$$

then

$$
\tilde{f}_{h} \rightarrow f \circ v \text { in } L_{\mathrm{loc}}^{1}(\mathbf{R} \times[0, T]) \text { as } h \rightarrow 0,
$$

where $f(c)=f_{l}(\cdots, c, c, c, \cdots)$. 
Proof. Let $I$ be a bounded interval in $\mathbf{R}$, and let $K=I \times[0, T]$. Then

$$
\left\|\tilde{f}_{h}-f(v)\right\|_{L^{1}(K)} \leqslant\left\|\tilde{f}_{h}-f\left(\tilde{V}_{h}\right)\right\|_{L^{1}(K)}+\left\|f\left(\tilde{V}_{h}\right)-f(v)\right\|_{L^{1}(K)} .
$$

The second term on the right vanishes as $h$ tends to zero since $f$ is Lipschitz continuous and $\tilde{V}_{h} \rightarrow v$ in $L_{\text {loc }}^{1}(\mathbf{R} \times[0, T])$. The first term can be bounded by

$$
\begin{aligned}
\left\|\tilde{f}_{h}-f\left(\tilde{V}_{h}\right)\right\|_{L^{1}(K)} & =\sum_{n \Delta t \leqslant T} \sum_{i h \in I}\left|f_{l}\left(\sigma_{i}\left(V_{h}^{n}\right)\right)-f_{l}\left(\cdots V_{h i}^{n}, V_{h i}^{n}, \cdots\right)\right| h \Delta t \\
\leqslant & \sum_{n \Delta t \leqslant T} \sum_{i h \in I} \sum_{|j| \leqslant m} L_{j}\left|V_{h(i+j)}^{n}-V_{h i}^{n}\right| h \Delta t \\
& +\sum_{n \Delta t \leqslant T} \sum_{i h \in I} \sum_{|j|>m} L_{j} 2\left\|V_{h}\right\|_{L^{\infty}(\mathbf{Z})} h \Delta t .
\end{aligned}
$$

For any $\varepsilon>0$, a value of $m$ can be found so that $\sum_{|j|>m} L_{j}<\varepsilon$. For that value of $m$ the second term of (2.4) is less than $2 \varepsilon\left\|V_{h}\right\|_{L^{\infty}(\mathbf{Z})}|I| T$. The first term can be bounded by

$$
\begin{aligned}
\sum_{n \Delta t \leqslant T} \sum_{|, j| \leqslant m} L_{j} & \sum_{i \in \mathbf{Z}}\left|V_{h(i+j)}^{n}-V_{h i}^{n}\right| h \Delta t \\
& \leqslant \sum_{n \Delta t \leqslant T} \sum_{|j| \leqslant m}|j| L_{j} \sum_{i \in \mathbf{Z}}\left|V_{h(i+1)}^{n}-V_{h i}^{n}\right| h \Delta t \leqslant\left(\sum_{|j| \leqslant m}|j| L_{j}\right) C T h .
\end{aligned}
$$

The latter bound can be made as small as necessary by picking $h$ small enough.

The proof of this lemma yields the following corollary.

COROLlaRY. If $\sum_{j \in \mathbf{z}}|j| L_{j}<\infty$, and $K=I \times[0, T]$, then

$$
\left\|\tilde{f}_{h}-f(v)\right\|_{L^{1}(K)} \leqslant\|f\|_{\text {Lip }} \cdot\left\|v-\tilde{V}_{h}\right\|_{L^{1}(K)}+\left(\sum_{j \in \mathbf{Z}}|j| L_{j}\right)\left|V_{h}^{n}\right|_{B V(\mathbf{Z})} T \cdot h .
$$

Proof of Theorem 2.2. The proof of the first statement of the theorem is similar to that in, for example, Crandall and Majda [4], or Sanders [22], and will not be repeated here. For example, one uses the bounds (b) and (c) of Theorem 2.1 to show that the functions $\tilde{U}_{h}$ are uniformly bounded in $B V(\mathbf{R} \times[0, T])$, hence are precompact in $L_{\text {loc }}^{1}(\mathbf{R} \times[0, T])$. Thus, a subsequence converges, as $h$ tends to zero, to a function $u$. Using Lemma 2.3 to handle the nonlinear term, one then proves in the usual way that the limit function is a weak solution of $(C)$, and that it satisfies the entropy condition (and hence is unique). None of this requires the use of the extra hypothesis (2.3). We will focus on the last statement of the theorem.

Crandall and Majda showed how one naturally gets an approximate (or numerical) entropy condition for the numerical method (2.2); we repeat that construction here. Extend the mapping $H$ of Theorem 2.1 to a map $\Lambda$ taking $B V(\mathbf{Z} \times[0, N])$ to itself by

$$
\Lambda(U)_{i}^{n}=H\left(U^{n}\right)_{i}
$$

If $C$ is a constant function in $B V(\mathbf{Z} \times[0, N])$, parts (c) and (e) of Theorem 2.1 imply

$\Lambda(U) \vee \Lambda(C) \leqslant \Lambda(U \vee C), \quad \Lambda(U) \wedge \Lambda(C) \geqslant \Lambda(U \wedge C)$, and $\Lambda(C)=C$, 
where $a \vee b=\max (a, b)$ and $a \wedge b=\min (a, b)$. The defining relation (2.2) for $U^{n+1}$ then implies that

$$
\begin{aligned}
& \frac{(\Lambda(U) \vee C-\Lambda(U) \wedge C)-(U \vee C-U \wedge C)}{\Delta t} \\
& \left.+\frac{1}{h}\left\{\begin{array}{c}
{\left[F_{1}(\sigma \Lambda(U \vee C))-F_{1}(\sigma \Lambda(U \wedge C))\right]-\left[F_{1}(\Lambda(U \vee C))-F_{1}(\Lambda(U \wedge C))\right]} \\
+\left[F_{2}(\sigma(U \vee C))-F_{2}(\sigma(U \wedge C))\right]-\left[F_{2}(U \vee C)-F_{2}(U \wedge C)\right]
\end{array}\right\}\right) \\
& =\frac{\Lambda(U) \vee C-\Lambda(U \vee C)}{\Delta t}-\frac{\Lambda(U) \wedge C-\Lambda(U \wedge C)}{\Delta t} \leqslant 0 .
\end{aligned}
$$

This inequality is a numerical entropy condition, and will be used in the sequel to bound the quantity $-\Lambda_{t}^{\varepsilon_{0}, \varepsilon}$.

For the most part, our proof of the error estimate reproduces the structure of Sander's Theorem IV [22].

Note that, if $\|\omega\|_{L_{1}^{1}\left(\mathbf{R}^{2}\right)}=\int_{\mathbf{R}^{2}}\left(\left|\omega_{x}(x, t)\right|+\left|\omega_{t}(x, t)\right|\right) d x d t$, then

$$
\|\omega(x+h, t+\tau)-\omega(x, t)\|_{L^{1}\left(\mathbf{R}^{2}\right)} \leqslant(|h|+|\tau|)\|\omega\|_{L_{1}^{1}\left(\mathbf{R}^{2}\right)} .
$$

(See [22].)

Following Sanders, for any number $g$ define the vector

$$
\tilde{F}_{g}\left(U^{n}\right)=F_{1}\left(U^{n+1} \vee G\right)+F_{2}\left(U^{n} \vee G\right)-\left(F_{1}\left(U^{n+1} \wedge G\right)+F_{2}\left(U^{n} \wedge G\right)\right),
$$

where $G=(\ldots, g, g, g, \ldots)$. We also define the real-valued function $F_{g}(w)=$ $\operatorname{sgn}(w-g)(f(w)-f(g))$ and the difference operators $\Delta_{t}^{+} V_{j}^{n}=V_{j}^{n+1}-V_{j}^{n}$ and $\Delta_{x}^{+} V_{j}^{n}=V_{j+1}^{n}-V_{j}^{n}$.

The conditions of Theorem 1.2 need to be verified. The approximation $\tilde{U}_{h}$ can obviously be taken to be right continuous, and Theorem 2.1 shows that

$$
\sup _{\left\{t^{\prime} \cdot|\tau|<\varepsilon_{0} \mid-t^{\prime}<\tau<t-t^{\prime}\right\}}\left\|\tilde{U}_{h}\left(t^{\prime}+\tau\right)-\tilde{U}_{h}\left(t^{\prime}\right)\right\|_{L^{1}(\mathbf{R})} \leqslant 2 L\left(\varepsilon_{0}+\Delta t\right)\left|u_{0}\right|_{B V(\mathbf{R})}
$$

Also, $\left\|U^{0}-u_{0}\right\|_{L^{1}(\mathbf{R})} \leqslant h\left|u_{0}\right|_{B V(\mathbf{R})}$. Therefore, it is necessary only to bound $-\Lambda_{t}^{\varepsilon_{0}, \varepsilon}$ when $v=\tilde{U}_{h}$. We assume $t=N \Delta t$.

Because $\tilde{U}_{h}$ is piecewise constant, $-\Lambda_{t}^{\varepsilon_{0}, \varepsilon}$ equals

$$
\begin{gathered}
-\int_{\mathbf{R} \times[0, t]} \sum_{\substack{n=0 \\
j \in \mathbf{Z}}}^{N-1} \int_{l_{i}^{\prime \prime}}\left[\left|U_{j}^{n}-u\left(x^{\prime}, t^{\prime}\right)\right| \frac{\partial}{\partial t^{\prime \prime}} \omega^{\prime \prime}\left(x^{\prime \prime}-x^{\prime}, t^{\prime \prime}-t^{\prime}\right)\right. \\
\left.+F_{u\left(x^{\prime}, t^{\prime}\right)}\left(U_{j}^{n}\right) \frac{\partial}{\partial x^{\prime \prime}} \omega\left(x^{\prime \prime}-x^{\prime}, t^{\prime \prime}-t^{\prime}\right)\right] d x^{\prime \prime} d t^{\prime \prime} d x^{\prime} d t^{\prime} \\
-\int_{\mathbf{R} \times[0, t]} \sum_{j} \int_{(j-1 / 2) h}^{(j+1 / 2) h}\left[\left|U_{j}^{0}-u\left(x^{\prime}, t^{\prime}\right)\right| \omega\left(x^{\prime \prime}-x^{\prime}, 0-t^{\prime}\right)\right. \\
\left.-\left|U_{j}^{N-1}-u\left(x^{\prime}, t^{\prime}\right)\right| \omega\left(x^{\prime \prime}-x^{\prime}, t-t^{\prime}\right)\right] d x^{\prime \prime} d x^{\prime} d t^{\prime},
\end{gathered}
$$

where $I_{j}^{n}=((j-1 / 2) h,(j+1 / 2) h) \times\left(t^{n}, t^{n+1}\right)$. Because of the special form of $\omega$, summing by parts and applying the integral version of the mean-value theorem 
transforms this integral to

$$
\begin{aligned}
& \int_{\mathbf{R} \times[0, t]} \sum_{\substack{n=0 \\
j \in \mathbf{Z}}}^{N-1}\left[\Delta_{t}^{+}\left|U_{j}^{n}-u\left(x^{\prime}, t^{\prime}\right)\right| \omega\left(\xi_{j}-x^{\prime}, t^{n+1}-t^{\prime}\right) h\right. \\
& \left.\quad+\Delta_{x}^{+} F_{u\left(x^{\prime}, t^{\prime}\right)}\left(U_{j}^{n}\right) \omega\left(x_{j+1}-x^{\prime}, \tau^{n}-t^{\prime}\right)\right] \Delta t d x^{\prime} d t^{\prime} \\
& -\int_{\mathbf{R} \times[0, t]} \sum_{j} \int_{(j-1 / 2) h}^{(j+1 / 2) h}\left[\left|U_{j}^{N}-u\left(x^{\prime}, t^{\prime}\right)\right|\right. \\
& \left.-\left|U_{j}^{N-1}-u\left(x^{\prime}, t^{\prime}\right)\right|\right] \omega\left(x^{\prime \prime}-x^{\prime}, t-t^{\prime}\right) d x^{\prime \prime} d x^{\prime} d t^{\prime}
\end{aligned}
$$

for some $\left(\xi_{j}, \tau^{n}\right) \in I_{j}^{n}$. By Theorem 2.1, the boundary term is less than $2 L \Delta t\left|u_{0}\right|_{B V(\mathbf{R})}$. Adding and subtracting $h \Delta_{t}^{+}\left|U_{j}^{n}-u\left(x^{\prime}, t^{\prime}\right)\right| \omega\left(x_{j+1}-x^{\prime}, \tau^{n}-t^{\prime}\right)$ in each term of the first sum yields

$$
\begin{gathered}
\int_{\mathbf{R} \times[0, t]} \sum_{\substack{n=0 \\
j \in \mathbf{Z}}}^{N-1} h\left[\Delta _ { t } ^ { + } | U _ { j } ^ { n } - u ( x ^ { \prime } , t ^ { \prime } ) | \left(\omega\left(\xi_{j}-x^{\prime}, t^{n+1}-t^{\prime}\right)\right.\right. \\
\left.\left.-\omega\left(x_{j+1}-x^{\prime}, \tau^{n}-t^{\prime}\right)\right)\right] d x^{\prime} d t^{\prime} \\
+\int_{\mathbf{R} \times[0, t]} \sum_{\substack{n=0 \\
j \in \mathbf{Z}}}^{N-1}\left[\Delta_{t}^{+}\left|U_{j}^{n}-u\left(x^{\prime}, t^{\prime}\right)\right| h\right. \\
\left.\quad+\Delta_{x}^{+} F_{u\left(x^{\prime}, t^{\prime}\right)}\left(U_{j}^{n}\right) \Delta t\right] \omega\left(x_{j+1}-x^{\prime}, \tau^{n}-t^{\prime}\right) d x^{\prime} d t^{\prime}
\end{gathered}
$$

The triangle inequality bounds the first of the two terms of the sum by

$$
\sum_{\substack{n=0 \\ j \in \mathbf{Z}}}^{N-1}\left|\Delta_{t}^{+} U_{j}^{n}\right| h \int_{\mathbf{R} \times[0, t]}\left|\omega\left(\xi_{j}-x^{\prime}, t^{n+1}-t^{\prime}\right)-\omega\left(x_{j+1}-x^{\prime}, \tau^{n}-t^{\prime}\right)\right| d x^{\prime} d t^{\prime}
$$

by part (c) of Theorem 2.1 and inequality (2.6), this term can now be bounded by

$$
L\left|u_{0}\right|_{B V(\mathbf{R})}(t+h) \cdot 2 h\|\omega\|_{L_{1}^{1}\left(\mathbf{R}^{2}\right)}
$$

To bound the second term, add and subtract $\Delta_{x}^{+} \tilde{F}_{u\left(x^{\prime}, t^{\prime}\right)}\left(U^{n}\right)_{j} \omega\left(x_{j+1}-x^{\prime}, \tau^{n}-t^{\prime}\right)$ in each term in the sum. The second part of (2.7) then becomes

$$
\begin{aligned}
& \int_{\mathbf{R} \times[0, t]} \sum_{\substack{n=0 \\
j \in \mathbf{Z}}}^{N-1}\left[\Delta_{t}^{+}\left|U_{j}^{n}-u\left(x^{\prime}, t^{\prime}\right)\right| h\right. \\
& \left.+\Delta_{x}^{+} \tilde{F}_{u\left(x^{\prime}, t^{\prime}\right)}\left(U^{n}\right)_{j} \Delta t\right] \omega\left(x_{j+1}-x^{\prime}, \tau^{n}-t^{\prime}\right) d x^{\prime} d t^{\prime} \\
& +\int_{\mathbf{R} \times[0, t]} \sum_{\substack{n=0 \\
j \in \mathbf{Z}}}^{N-1} \Delta t \Delta_{x}^{+}\left(F_{u\left(x^{\prime}, t^{\prime}\right)}\left(U_{j}^{n}\right)\right. \\
& \left.\quad-\tilde{F}_{u\left(x^{\prime}, t^{\prime}\right)}\left(U^{n}\right)_{j}\right) \omega\left(x_{j+1}-x^{\prime}, \tau^{n}-t^{\prime}\right) d x^{\prime} d t^{\prime} .
\end{aligned}
$$


The numerical entropy condition (2.5) shows that the first term above is nonpositive. The second term of (2.8) can be bounded by

$$
\begin{gathered}
\int_{\mathbf{R} \times[0, t]} \sum_{\substack{n=0 \\
j \in \mathbf{Z}}}^{N-1} \Delta t\left|F_{u\left(x^{\prime}, t^{\prime}\right)}\left(U_{j}^{n}\right)-\tilde{F}_{u\left(x^{\prime}, t^{\prime}\right)}\left(U^{n}\right)_{j}\right| \\
\cdot\left|\omega\left(x_{j+1}-x^{\prime}, \tau^{n}-t^{\prime}\right)-\omega\left(x_{j}-x^{\prime}, \tau^{n}-t^{\prime}\right)\right| d x^{\prime} d t^{\prime} \\
\leqslant 2 h\|\omega\|_{L_{1}^{1}\left(\mathbf{R}^{2}\right)} \sum_{\substack{n=0 \\
j \in \mathbf{Z}}}^{N-1} \Delta t\left|F_{g}\left(U_{j}^{n}\right)-\tilde{F}_{g}\left(U^{n}\right)_{j}\right|
\end{gathered}
$$

for any $g$. To complete the theorem, one must show that there exists a constant $K$, independent of $h, \Delta t$, and $n$ such that

$$
\sum_{j \in \mathbf{Z}}\left|\tilde{F}_{g}\left(U^{n}\right)_{j}-F_{g}\left(U_{j}^{n}\right)\right| \leqslant K\left|u_{0}\right|_{B V(\mathbf{R})} \cdot
$$

Let us examine the first of the four constituent parts in the definition of $\tilde{F}_{g}$. Lemma 2.3, its following corollary, and Assumption 2 yield

$$
\begin{aligned}
& \sum_{j \in \mathbf{Z}}\left|F_{2}\left(U^{n} \vee G\right)_{j}-f_{2}\left(\ldots, U_{j}^{n} \vee g, U_{j}^{n} \vee g, \ldots\right)\right| \\
& \leqslant M\left|U^{n} \vee G\right|_{B V(\mathbf{Z})} \leqslant M\left|u_{0}\right|_{B V(\mathbf{R}) .}
\end{aligned}
$$

The additional use of part (c) of Theorem 2.1 yields

$$
\begin{aligned}
& \sum_{j \in \mathbf{Z}}\left|F_{1}\left(U^{n+1} \vee G\right)_{j}-f_{1}\left(U_{j}^{n} \vee g\right)\right| \\
& \leqslant \sum_{j \in \mathbf{Z}}\left|F_{1}\left(U^{n+1} \vee G\right)_{j}-f_{1}\left(\ldots, U_{j}^{n+1} \vee g, U_{j}^{n+1} \vee g, \ldots\right)\right| \\
& \quad+\sum_{j \in \mathbf{Z}}\left|f_{1}\left(\ldots, U_{j}^{n+1} \vee g, U_{j}^{n+1} \vee g, \ldots\right)-f_{1}\left(\ldots, U_{j}^{n} \vee g, U_{j}^{n} \vee g, \ldots\right)\right| \\
& \leqslant\left(2 L \frac{\Delta t}{h}\left\|f_{1}\right\|_{\text {Lip }}+M\right)\left|u_{0}\right|_{B V(\mathbf{R})} .
\end{aligned}
$$

The other two terms can be handled similarly. Summing the four inequalities for the four parts of $\tilde{F}$ yields (2.9).

We have bounded $-\Lambda_{t}^{\varepsilon_{0}, \varepsilon}$ by $\left(C_{1} h+C_{2} \Delta t\right)\left|u_{0}\right|_{B V(\mathbf{R})}\|\omega\|_{L_{1}^{1}\left(\mathbf{R}^{2}\right)}$. Since $\|\omega\|_{L_{1}^{1}\left(\mathbf{R}^{2}\right)} \leqslant$ $C\left(\varepsilon_{0}^{-1}+\varepsilon^{-1}\right)$, the theorem follows by setting $\varepsilon=\varepsilon_{0}=h^{1 / 2}$.

Numerical evidence suggests that the inequality (2.3) is necessary to obtain a convergence rate of order $h^{1 / 2}$. Consider the linear problem

$$
\begin{gathered}
u_{t}+u_{x}=0, \quad x \in \mathbf{R}, t>0, \\
u(x, 0)=\chi_{(0, \infty)}(x), \quad x \in \mathbf{R},
\end{gathered}
$$

and numerical fluxes $f_{1}(U)=0$ and

$$
f_{2}(U)=C_{1} \sum_{j \leqslant 0}|j|^{-3 / 2} U_{j}
$$

where $C_{1}$ is chosen so that the method is consistent. If $\Delta t / h$ is chosen so that a Courant-Friedrichs-Lewy condition holds (see the next section), then this method satisfies Assumptions 1 through 4, but not (2.3). When this method is implemented, 
a convergence rate of about $h^{1 / 3}$ is observed. Thus, it seems that numerical methods that satisfy Assumptions 1 through 4 but do not satisfy (2.3) have the dubious distinction of being the methods that converge to the entropy solution of the conservation law $(\mathrm{C})$ with the slowest known rates of convergence.

3. Dispersive Numerical Approximations to Conservation Laws. We now present the finite-difference approximations for $(\mathrm{C})$ that motivated the previous analysis. These are modeled on the Sobolev-type equation

$$
\begin{gathered}
u_{t}+f(u)_{x}-\nu g(u)_{x x}-\alpha^{2} u_{x x t}=0, \quad x \in \mathbf{R}, t>0, \\
u(x, 0)=u_{0}(x), \quad x \in \mathbf{R} .
\end{gathered}
$$

This equation, regularizing (C) by incorporating a dispersive term $\left(-\alpha^{2} u_{x x t}\right)$ as well as a term simulating nonlinear dissipation $\left(-\nu g(u)_{x x}\right)$, has been used to model the passage of small-amplitude shallow-water waves (see Benjamin et al. [1] and Bona et al. [2]). The value of $(\mathbf{S})$ as a regularization of $(C)$ is studied in another paper [20]. The numerical schemes based on (S) are very similar to those used by Douglas et al. [8].

We will use the following notation. $W=\left\{W_{i}^{n}\right\}_{i \in \mathbf{Z}}^{n \in[0, N]}$ denotes a function defined on $\mathbf{Z} \times[0, N]$, where $N$ is fixed. The value of the function $W$ for the $n$th time step will be denoted by $W^{n}=\left\{W_{i}^{n}\right\}_{i \in \mathbf{z}}$. If $h$ and $\Delta t$ are two fixed positive numbers, define the divided differences

$$
\begin{gathered}
d_{x} W_{i}^{n}=\frac{W_{i+1}^{n}-W_{i-1}^{n}}{2 h}, \\
d_{x}^{2} W_{i}^{n}=\frac{W_{i-1}^{n}-2 W_{i}^{n}+W_{i+1}^{n}}{h^{2}}, \text { and } d_{t} W_{i}^{n}=\frac{W_{i}^{n+1}-W_{i}^{n}}{\Delta t} .
\end{gathered}
$$

The two specific difference schemes to be considered here are the method using forward differencing in time,

(3.1a) $\left(1-\alpha^{2} d_{x}^{2}\right) d_{t} U_{i}^{n}+d_{x} f\left(U^{n}\right)_{i}-\nu d_{x}^{2} g\left(U^{n}\right)_{i}=0, \quad n \geqslant 0, i \in \mathbf{Z}$,

and the backward-difference method,

(3.1b) $\left(1-\alpha^{2} d_{x}^{2}\right) d_{t} U_{i}^{n}+d_{x} f\left(U^{n+1}\right)_{i}-\nu d_{x}^{2} g\left(U^{n+1}\right)_{i}=0, \quad n \geqslant 0, i \in \mathbf{Z}$.

Each method uses the same initial value

$$
U_{i}^{0}=\frac{1}{h} \int_{(i-1 / 2) h}^{(i+1 / 2) h} u_{0}(x) d x,
$$

where $u_{0}$ is given in (C). (Any initial data such that $U^{0} \rightarrow u_{0}$ in $L^{1}(\mathbf{R})$ and $\left|U^{0}\right|_{B V(\mathbf{Z})}$ is bounded as $h \rightarrow 0$ would suffice.) These equations can be viewed as discretizations of $(\mathrm{S})$ using centered differences in space and either forward or backward differences in time.

These schemes can also be interpreted as "averaged" difference methods. We start with a centered-difference approximation to $f(u)_{x}$ and add a second-order dissipative term $-\nu d_{x}^{2} g(u)$ for stability. Before these values are used as the time difference of $u$, however, they are averaged using the operator $\left(1-\alpha^{2} d_{x}^{2}\right)^{-1}$. This is similar to a scheme used by A. Jameson to calculate steady-state solutions of the Euler equations for gas dynamics [14].

We first write these methods as $d_{t} U_{i}^{n}+A\left(U^{n}\right)_{i} / h=0$, or $d_{t} U_{i}^{n}+A\left(U^{n+1}\right)_{i} / h=$ 0 . Note that the discrete-difference operator $1-\alpha^{2} d_{x}^{2}$ may be inverted on $\mathbf{Z}$, subject to boundedness at infinity, by discrete convolution with the function $M_{\alpha}(j)=a K^{|j|}$. 
If $r=\alpha / h$, then

$$
K=\frac{1+2 r^{2}-\sqrt{1+4 r^{2}}}{2 r^{2}},
$$

the smaller root of the equation $r^{2} K^{2}-\left(1-2 r^{2}\right) K+r^{2}=0$, and $a=$ $\left(1+4 r^{2}\right)^{-1 / 2}$. Performing the inversion yields, in the forward-difference case,

$$
\begin{aligned}
d_{t} U_{i}^{n}= & a \sum_{l \in \mathbf{Z}} K^{|l-i|}\left\{-d_{x} f\left(U^{n}\right)_{l}+\nu d_{x}^{2} g\left(U^{n}\right)_{l}\right\} \\
= & a \sum_{l \in \mathbf{Z}} K^{|l-i|}\left\{\frac{f\left(U_{l-1}^{n}\right)+f\left(U_{l}^{n}\right)}{2 h}+\nu \frac{g\left(U_{l-1}^{n}\right)-g\left(U_{l}^{n}\right)}{h^{2}}\right\} \\
& -a \sum_{l \in \mathbf{Z}} K^{|l-i|}\left\{\frac{f\left(U_{l}^{n}\right)+f\left(U_{l+1}^{n}\right)}{2 h}+\nu \frac{g\left(U_{l}^{n}\right)-g\left(U_{l-1}^{n}\right)}{h^{2}}\right\} \\
= & a \sum_{l \neq i} K^{|l-i|}\left\{\operatorname{sgn}(i-l)\left(\frac{1}{K}-K\right) \frac{f\left(U_{l}^{n}\right)}{2 h}+\nu \frac{g\left(U_{l}^{n}\right)}{\alpha^{2}}\right\} \\
& -\nu(1-a) \frac{g\left(U_{i}^{n}\right)}{\alpha^{2}} .
\end{aligned}
$$

Formula (3.2b) is a simple rewriting of (3.2a). Formula (3.2c) is derived by gathering all terms depending on $U_{l}^{n}$ in one place (or by summation by parts).

Call the operator on the right-hand side of (3.2) $-A(U) / h$. This $A$ corresponds to $A_{1}$ and $A_{2}$ of the previous section. We now claim that under certain restrictions on $\alpha, g, h$, and $\Delta t$ the operator $A$ fulfills Assumptions 1 through 4.

For $l=1$ (the implicit case) and $l=2$ (the explicit case), let

$$
f_{l}(U)=a \sum_{i \in \mathbf{Z}} K^{|i|}\left\{\frac{f\left(U_{i-1}\right)+f\left(U_{i}\right)}{2}+\nu \frac{g\left(U_{i-1}\right)-g\left(U_{i}\right)}{h}\right\} .
$$

The function $f_{l}$ satisfies Assumption 1 with

$$
L_{i}=a\left(|K|^{|i|}+|K|^{|i+1|}\right)\left\{\frac{\|f\|_{\text {Lip }}}{2}+\frac{\nu\|g\|_{\text {Lip }}}{h}\right\}
$$

for $i \in \mathbf{Z}$, provided that the ratios $\alpha / h$ and $\nu / h$ are fixed as $h$ tends to zero. The function $f_{l}$ will then be independent of $h$, and will satisfy Assumption 1. It also satisfies the extra assumption (2.3) of Theorem 2.2.

As for Assumption 2,

$$
f_{l}(C)=a \sum_{i \in \mathbf{Z}} K^{|i|}\left\{\frac{f(c)+f(c)}{2}+\nu \frac{g(c)-g(c)}{h}\right\}=f(c),
$$

since $a \sum_{i \in \mathbf{Z}} K^{|i|}=1$.

The following theorem delineates when Assumptions 3 and 4 hold.

THEOREM 3.1. Assume that $f$ and $g$ are Lipschitz continuous. The mapping $H(U)=$ $U-\Delta t A(U) / h$ is order-preserving on $B V(\mathbf{Z})$ if and only if

(1) the function

$$
\xi-\frac{\Delta t(1-a)}{\alpha^{2}} \nu g(\xi)
$$


is nondecreasing on $\mathbf{R}$ (a Courant-Friedrichs-Lewy condition [21]), and

(2) the functions

$$
\nu g(\xi) \pm\left(\frac{h^{2}}{4}+\alpha^{2}\right)^{1 / 2} f(\xi)
$$

are nondecreasing on $\mathbf{R}$.

The mapping $A$ of (3.2) satisfies Assumption 3 if and only if (2) holds.

Proof. Clearly, the mapping $H(U)$ is order-preserving if and only if $H(U)_{i}$ is a nondecreasing function of $U_{j}$ for every $j$. Using expression (3.2c) for $-A(U) / h$, we see that $H(U)_{i}$ is a nondecreasing function of $U_{i}$ if and only if $\xi-$ $\nu \Delta t(1-a) g(\xi) / \alpha^{2}$ is nondecreasing on $\mathbf{R}$, which is condition (1). For $j \neq i$, $H(U)_{i}$ is a nondecreasing function of $U_{j}$ if and only if the functions

$$
\nu \frac{g(\xi)}{\alpha^{2}} \pm\left(\frac{1}{K}-K\right) \frac{f(\xi)}{2 h}
$$

are nondecreasing on $\mathbf{R}$, the sign depending on $\operatorname{sgn}(i-j)$. Condition (2) may be derived from (3.5) by using the definition of $K$.

We now prove the last claim. For any $U, V \in B V(\mathbf{Z})$, with $U-V \in L^{1}(\mathbf{Z})$, multiplying (3.2c) by $\operatorname{sgn}\left(U_{i}-V_{i}\right)$ gives

$$
\begin{aligned}
(A(U) & -A(V))_{i} \operatorname{sgn}\left(U_{i}-V_{i}\right) \\
\geqslant & -h a \sum_{l \neq i} K^{|l-i|}\left|\operatorname{sgn}(i-l)\left(\frac{1}{K}-K\right) \frac{f\left(U_{l}\right)-f\left(V_{l}\right)}{2 h}+\nu \frac{g\left(U_{l}\right)-g\left(V_{l}\right)}{\alpha^{2}}\right| \\
& +h \nu \frac{1-a}{\alpha^{2}}\left|g\left(U_{i}\right)-g\left(V_{i}\right)\right| .
\end{aligned}
$$

Since $U-V$ is in $L^{1}(\mathbf{Z})$, both terms of the right-hand side of (3.6) are in $L^{1}(\mathbf{Z})$. Sum (3.6) over $i \in \mathbf{Z}$ and change the order of summation. Since $a \sum_{l=1}^{\infty} K^{\prime}=(1-a) / 2$, this yields

$$
\begin{aligned}
\sum_{i \in \mathbf{Z}}(A(U)-A(V))_{i} \operatorname{sgn}\left(U_{i}-V_{i}\right) & \\
\geqslant-\left(\frac{1-a}{2}\right) h \sum_{l \in \mathbf{Z}}\{\mid & -\left(\frac{1}{K}-K\right) \frac{f\left(U_{l}\right)-f\left(V_{l}\right)}{2 h}+\nu \frac{g\left(U_{l}\right)-g\left(V_{l}\right)}{\alpha^{2}} \mid \\
& \left.+\left|\left(\frac{1}{K}-K\right) \frac{f\left(U_{l}\right)-f\left(V_{l}\right)}{2 h}+\nu \frac{g\left(U_{l}\right)-g\left(V_{l}\right)}{\alpha^{2}}\right|\right\} \\
& +(1-a) h \nu \sum_{i \in \mathbf{Z}} \frac{\left|g\left(U_{i}\right)-g\left(V_{i}\right)\right|}{\alpha^{2}} .
\end{aligned}
$$

Because of condition (2), for any $\xi, \eta \in \mathbf{R}$,

$$
\begin{aligned}
\frac{1}{2}\{\mid- & \left(\frac{1}{K}-K\right) \frac{f(\xi)-f(\eta)}{2 h}+\nu \frac{g(\xi)-g(\eta)}{\alpha^{2}} \mid \\
& \left.+\left|\left(\frac{1}{K}-K\right) \frac{f(\xi)-f(\eta)}{2 h}+\nu \frac{g(\xi)-g(\eta)}{\alpha^{2}}\right|\right\} \\
& =\nu \frac{|g(\xi)-g(\eta)|}{\alpha^{2}}
\end{aligned}
$$


and so

$$
\sum_{i \in \mathbf{Z}}(A(U)-A(V))_{i} \operatorname{sgn}\left(U_{i}-V_{i}\right) \geqslant 0 .
$$

Thus, $A$ satisfies Assumption 3.

If (2) does not hold, then there is an $\eta>\xi$ such that one of

$$
\left\{ \pm\left(\frac{1}{K}-K\right) \frac{f(\eta)}{2 h}+\frac{g(\eta)}{\alpha^{2}}\right\}-\left\{ \pm\left(\frac{1}{K}-K\right) \frac{f(\xi)}{2 h}+\frac{g(\xi)}{\alpha^{2}}\right\}=-\delta<0 .
$$

If $U^{0}=\eta e^{0}, V^{0}=\xi e^{0}, U^{1}=U^{0}-\Delta t A\left(U^{0}\right) / h$, and $V^{1}$ is defined similarly, a direct calculation shows that

$$
\left\|V^{1}-U^{1}\right\|_{L^{1}(\mathbf{z})} \geqslant\left\|U^{0}-V^{0}\right\|_{L^{1}(\mathbf{z})}+C \Delta t \delta,
$$

for some $C$.

Since $A$ is Lipschitz continuous on $L^{1}(\mathbf{Z})$, there exists another constant $C$ such that, if $\tilde{U}^{0}=U^{1}+\Delta t A\left(U^{1}\right) / h$, then

$$
\left\|U^{0}-\tilde{U}^{0}\right\|_{L^{1}(\mathbf{Z})} \leqslant C\|A\|_{\text {Lip }}^{2}\left\|U^{0}\right\|_{L^{1}(\mathbf{Z})} \Delta t^{2} .
$$

Hence, for some value of $\Delta t$, we have

$$
\left\|U^{1}-V^{1}\right\|_{L^{1}(\mathbf{Z})}>\left\|\tilde{U}^{0}-\tilde{V}^{0}\right\|_{L^{1}(\mathbf{Z})} .
$$

Therefore, the solution operator of the backward-difference equation is not a contraction on $L^{1}(\mathbf{Z})$, so the mapping $A$ does not fulfill Assumption 3.

When $f$ and $g$ are differentiable, (3.4) is equivalent to

$$
\nu g^{\prime}(\xi) \geqslant\left(\frac{h^{2}}{4}+\alpha^{2}\right)^{1 / 2}\left|f^{\prime}(\xi)\right| \text { for all } \xi \in \mathbf{R} .
$$

When $h$ is 0 , this condition is necessary and sufficient for the differential equation

$$
u_{t}+f(u)_{x}-\nu g(u)_{x x}-\alpha^{2} u_{x x t}=0
$$

to satisfy a maximum principle (see [20]). When $\alpha$ is $0,(3.8)$ reduces to

$$
\nu g^{\prime}(\xi) \geqslant \frac{h}{2}\left|f^{\prime}(\xi)\right|
$$

which is the necessary and sufficient condition for the solution operator of the difference equation

$$
d_{t} U^{n}+d_{x} f\left(U^{n+1}\right)-\nu d_{x}^{2} g\left(U^{n+1}\right)=0
$$

to be order-preserving (cf. Douglas [7] and Crandall and Majda [4]).

For a given level of dispersion and a given transport term $f$, the inequality (3.8) may be used to find a function $g$ such that the equation (3.2) has the least amount of artificial diffusion necessary for Assumptions 1 through 4 to hold. In this case

$$
\nu g^{\prime}(\xi)=\left(\frac{h^{2}}{4}+\alpha^{2}\right)^{1 / 2}\left|f^{\prime}(\xi)\right| \text {. }
$$

With this $g$, if $f$ is nondecreasing, then $U_{i}^{n+1}-U_{i}^{n}$ cispends only on the value of $U_{j}^{n}$ for $j \leqslant i$; that is, it is a one-sided, upwind, difference method. It is in this sense that we call the methods investigated here generalized upwind-difference methods. We note that the generalized upwind-difference scheme of Engquist and Osher [10] can 
be characterized as the scheme of the form (3.10) with the least amount of dissipation necessary for stability.

If $g$ is chosen so that equality holds in (3.8), then condition (3.3) is equivalent to

$$
\left|f^{\prime}(\xi)\right| \frac{\Delta t}{h} \leqslant\left(\frac{1}{4}+\frac{\alpha^{2}}{h^{2}}\right)^{1 / 2}+\frac{1}{2} .
$$

Larger time steps may be taken whenever $\alpha$ is positive. The effective width of the kernel $M_{\alpha}$ is of order $r=\alpha / h$ meshpoints. Equation (3.11) can be interpreted as saying that $\Delta t$ may be increased so long as the characteristics coming into the point $(i h,(n+1) \Delta t)$ start within the effective width of the spatial-difference operator centered at $(i h, n \Delta t)$.

Douglas et al. [8] have pointed out that an implicit finite-element method based on the regularization (S) may be used to approximate $(C)$. Define the space $M_{h}=\left\{v \in C^{0}(\mathbf{R})|v|_{[i h,(i+1) h]}\right.$ is a linear function $\} \cap L^{2}(\mathbf{R})$ and the inner product $(v, w)=\int_{\mathbf{R}} v(x) w(x) d x$. We look for a sequence of functions $\left\{w^{0}, w^{1}, \ldots\right\}$ such that for all $v \in M_{h}$

$$
\left(\frac{w^{n}-w^{n-1}}{\Delta t}, v\right)-\left(f\left(w^{n}\right), v_{x}\right)+\nu\left(g\left(w^{n}\right)_{x}, v_{x}\right)+\alpha^{2}\left(\frac{w_{x}^{n}-w_{x}^{n-1}}{\Delta t}, v_{x}\right)=0 .
$$

Here $w^{0}$ is some suitably chosen initial value. If the integrals are evaluated with the trapezoid rule, then the algebraic equations to be solved are exactly of the form (3.2b), with

$$
\alpha_{\text {finite element }}^{2}=\alpha_{\text {finite difference }}^{2}+\frac{1}{6} h^{2} .
$$

As they note in [8], the introduction of explicit dispersion unifies the finite-difference and finite-element approach to the problem. For the solution operator of the finite-element problem to be a contraction in $L^{1}(\mathbf{R})$ and satisfy a maximum principle, it is necessary that $\alpha^{2} \geqslant \frac{1}{6} h^{2}$, so that the corresponding finite-difference $\alpha^{2}$ is positive, and

$$
\nu g^{\prime}(\xi) \geqslant\left(\frac{1}{12} h^{2}+\alpha^{2}\right)^{1 / 2}\left|f^{\prime}(\xi)\right|
$$

for all $\xi \in \mathbf{R}$. This may be compared with (3.8) and (3.9). If these conditions are satisfied, then the finite-element methods fall into the class of methods studied in this paper.

Requirement (3.8) depends on the level of dispersion $\alpha$ and the discretization parameter $h$ only through the combination $h^{2} / 4+\alpha^{2}$. If the dispersion and discretization are altered, but this expression remains fixed, then the same amount of dissipation is necessary for stability. In this sense, the effects of spatial discretization and artificial dispersion are interchangeable when "working against" a maximum principle. Thus, we may be led to the view that condition (3.9) is required by the dispersion introduced by discretizing the purely dissipative equation

$$
u_{t}+f(u)_{x}-\nu g(u)_{x x}=0
$$

to obtain (3.10). Trefethen [23] has written a survey on the effects of dispersion on numerical schemes. 
This view may be investigated further by considering formally the following difference problem, which is continuous in time:

$$
\begin{aligned}
\frac{d}{d t} u(x, t)+\frac{f(u(x+h, t))-f(u(x-h, t))}{2 h} & \\
-\nu \frac{g(u(x-h, t))-2 g(u(x, t))+g(u(x+h, t))}{h^{2}} & =0 .
\end{aligned}
$$

Here it is assumed that $f$ and $g$ are smooth, that $\nu / h$ is fixed, and that there is a smooth solution $u$ of (3.12). We follow the lead of many others in expanding $f(u)$ and $g(u)$ in terms of their Taylor series expansions about $x$ to yield

$$
u_{t}+f(u)_{x}-\nu g(u)_{x x}+\frac{h^{2}}{6} f(u)_{x x x}=0
$$

to order $h^{3}$. From this equation, $u_{t}=-f(u)_{x}+O(h)$, so that, again to order $h^{3}$,

$$
u_{t}+f(u)_{x}-\nu g(u)_{x x}-\frac{h^{2}}{6} u_{x x t}=0 .
$$

This partial differential equation, called a modified equation [13] is associated naturally with the finite-difference equation (3.12). It is a differential equation modeling a difference equation. It is also a Sobolev equation of the type introduced and studied in [20]. For the solutions of this equation to satisfy a maximum principle, it is necessary and sufficient that for all $\xi \in \mathbf{R}$

$$
\nu g^{\prime}(\xi) \geqslant \frac{h}{\sqrt{6}}\left|f^{\prime}(\xi)\right| \text {. }
$$

This inequality, although not exactly the same as (3.9), is so similar that it might explain heuristically why a condition such as (3.9) is necessary for the solutions of the finite-difference method (3.10) to satisfy a maximum principle.

Department of Mathematics

Purdue University

West Lafayette, Indiana 47907

1. T. B. Benjamin, J. L. Bona \& J. J. Mahony, "Model equations for long waves in nonlinear dispersive systems,” Philos. Trans. Roy. Soc. London Ser. A, v. 272, 1972, pp. 47-78.

2. J. L. Bona, W. G. Pritchard \& L. R. Scott, "An evaluation of a model equation for water waves," Philos. Trans. Rov. Soc. London Ser. A, v. 302, 1981, pp. 457-510.

3. M. G. Crandall \& T. M. Liggett, "Generation of semi-groups of nonlinear transformations on general Banach spaces," Amer. J. Math., v. 93, 1971, pp. 265-298.

4. M. G. Crandall \& A. Majda, "Monotone difference approximations for scalar conservation laws," Math. Comp., v. 34, 1980, pp. 1-21.

5. M. G. Crandall \& L. Tartar, "Some relations between nonexpansive and order preserving mappings," Proc. Amer. Math. Soc., v. 78, 1980, pp. 385-390.

6. K. Deimling, Ordinary Differential Equations in Banach Spaces, Springer-Verlag, New York, 1977.

7. J. Douglas, JR., "Simulation of a linear waterflood," in Free Boundary Problems, Proceedings of a seminar held in Pavia, Sept.-Oct. 1979, Vol. II, Istituto Nazionale di Alta Matematica "Francesco Severi," Roma, 1980.

8. J. Douglas, JR., R. P. Kendall \& M. F. Wheeler, "Long wave regularization of one-dimensional, two-phase, immiscible flow in porous media," Finite Element Methods for Convection Dominated Flows, AMD-v. 34, ASME, New York, 1979, pp. 201-211.

9. J. Douglas, JR. \& M. F. Wheeler, "Implicit, time-dependent variable grid finite difference methods for the approximation of a linear waterflood," Math. Comp., v. 40, 1983, pp. 107-122.

10. B. ENGQUiST \& S. OSHER, "Stable and entropy satisfying approximations for transonic flow calculations," Math. Comp., v. 34, 1980, pp. 45-75. 
11. E. Giustr, Minimal Surfaces and Functions of Bounded Variation, Australian National University, 1977

12. A. Harten, J. M. Hyman \& P. D. Lax, "On finite difference approximations and entropy conditions for shocks," Comm. Pure Appl. Math., v. 29, 1976, pp. 297-322.

13. (j. W. Hedstrom, "Models of difference schemes for $u_{t}+u_{x}=0$ by partial differential equations," Math. Comp., v. 29, 1975, pp. 969-977.

14. A. JAMESON \& T. J. BAKER, Solution of the Euler Equations for Complex Configurations, AIAA paper 83-1929. 1983.

15. S. N. KRUzhKov, "First order quasilinear equations with several independent variables," Math. USSR Sh.. v. 10, 1970, pp. 217-243.

16. N. N. KUZNETSOV, "Accuracy of some approximate methods for computing the weak solutions of a first-order quasi-linear equation," USSR Comput. Math. and Math. Phys., v. 16, no. 6, 1976, pp. 105-119.

17. N. N. KuZnetsov \& S. A. Voloshin, "On the stability of a class of implicit finite-difference schemes," Dokl. Akad. Nauk SSSR, v. 242, no. 3, 1978, pp. 525-528.

18. P. D. Lax \& B. Wendroff, "Systems of conservation laws," Comm. Pure Appl. Math., v. 13, 1960, pp. $217-237$.

19. B. J. LuCIER, Dispersive Approximations for Hyperbolic Conservation Laws, ANL-81-74, Argonne National Laboratory, 1981.

20. B. J. LuCIER, “On Sobolev regularizations of hyperbolic conservation laws," Comm. Partial Differential Equations, v. 10, no. 1, 1985, pp. 1-28.

21. R. D. Richtmyer \& K. W. Morton, Difference Methods for Initial-Value Problems, 2nd ed., Wiley, New York, 1967.

22. R. SANDERS, "On convergence of monotone finite difference schemes with variable spatial differencing," Math. Comp., v. 40, 1983, pp. 91-106.

23. L. N. TRefethen, "Group velocity in finite difference schemes," SIAM Rev., v. 24, 1982, pp. 113-136.

24. S. A. Voloshin, "On a class of monotonic finite difference approximations of a first-order quasi-linear equation," Dokl. Akad. Nauk SSSR, v. 242, no. 1, 1978, pp. 14-16.

25. S. A. Voloshin, "On a class of implicit finite-difference schemes," USSR Comput. Math. and Math. Phys., v. 23, no. 2, 1983, pp. 59-63.

26. G. B. Whitham, Linear and Nonlinear Waves, Wiley, New York, 1974. 